\title{
Supporting Information: Surface Adsorption from the Exchange-Hole Dipole Moment Dispersion Model
}

Table 1: Comparison of calculated cubic lattice parameters for the three metals, in $\AA$, with experimental results from R. Wyckoff, Crystal structures (Interscience publishers, New York, 1963).

\begin{tabular}{l|cc}
\hline & Calc & Exp. \\
\hline $\mathrm{Cu}$ & 3.573 & 3.615 \\
$\mathrm{Ag}$ & 4.080 & 4.086 \\
$\mathrm{Au}$ & 4.145 & 4.078 \\
\hline
\end{tabular}

Table 2: Calculated molecule-surface distances, in $\AA$, measured as the distance from the heteroatom (or carbon in the case of benzene) to the surface. The angles between the surface and the molecular aromatic moiety, in degrees, are also indicated.

\begin{tabular}{l|ccc|lcc}
\hline Molecule & \multicolumn{3}{|c|}{ Distances } & \multicolumn{3}{c}{ Angles } \\
& $\mathrm{Cu}$ & $\mathrm{Ag}$ & $\mathrm{Au}$ & $\mathrm{Cu}$ & $\mathrm{Ag}$ & $\mathrm{Au}$ \\
\hline Benzene & 2.71 & 3.03 & 3.15 & 0.9 & 4.3 & 3.2 \\
Furan & 2.99 & 3.27 & 3.33 & 5.2 & 6.6 & 6.8 \\
Furan perp. & 3.06 & 3.16 & 3.28 & 58.4 & 68.3 & 68.0 \\
Thiophene & 2.99 & 3.04 & 2.95 & 2.2 & 2.2 & 4.6 \\
Thiophene perp. & 2.38 & 2.77 & 3.23 & 82.0 & 57.5 & 85.5 \\
Thiophenol & 2.43 & 2.81 & 2.75 & 5.1 & 3.8 & 6.6 \\
Thiophenol perp. & 2.78 & 2.97 & 3.02 & 79.4 & 77.9 & 77.1 \\
Pyridine & 2.17 & 2.68 & 3.04 & 24.5 & 13.1 & 8.6 \\
Pyridine Perp. & 2.08 & 2.40 & 2.38 & 82.1 & 77.3 & 65.9 \\
1,4-Benzenediamine (cis) & 2.94 & 3.16 & 3.26 & 1.0 & 1.7 & 0.1 \\
1,4-Benzenediamine (trans) & 2.22 & 2.52 & 2.52 & 8.0 & 6.8 & 8.4 \\
\hline Guanine & 2.26 & 2.54 & 2.65 & 16.0 & 13.4 & 11.8 \\
Cytosine & 2.15 & 2.51 & 2.64 & 21.3 & 17.1 & 14.2 \\
Adenine & 3.02 & 3.09 & 3.18 & 0.8 & 2.0 & 1.4 \\
Thymine & 2.82 & 2.93 & 3.21 & 7.3 & 6.9 & 4.0 \\
\hline
\end{tabular}


Table 3: Convergence of the calculated adsorption energy of 1,4-benzenediamine (in the trans orientation) on the copper surface, with respect to changes in plane-wave cut-off $\left(E_{\text {cut }}\right)$ and k-point grid. All results are for the 4-layer surface. For comparison, the binding energy on a 5-layer surface with a $4 \times 4 \times 1 \mathrm{k}$-point grid and a 60 Ry cut-off is $1.2579 \mathrm{eV} / \mathrm{molecule.}$

\begin{tabular}{ccc}
\hline$E_{\text {cut }}(\mathrm{Ry})$ & k-points & $E_{a d s}(\mathrm{eV} / \mathrm{molec})$ \\
\hline 50 & $4 \times 4 \times 1$ & 1.2422 \\
60 & $4 \times 4 \times 1$ & 1.2429 \\
70 & $4 \times 4 \times 1$ & 1.2428 \\
80 & $4 \times 4 \times 1$ & 1.2420 \\
\hline 60 & $3 \times 3 \times 1$ & 1.2935 \\
60 & $4 \times 4 \times 1$ & 1.2429 \\
60 & $5 \times 5 \times 1$ & 1.2385 \\
60 & $6 \times 6 \times 1$ & 1.2401 \\
\hline
\end{tabular}

Table 4: Comparison of adsorption energies, in eV/molecule, for the cases where the isolatedmolecule calculations were performed using the same cell as in the physisorbed complexes or in a supercell surrounded in all directions by a large vacuum ("Vac.").

\begin{tabular}{lcccccc}
\hline Molecule & $\mathrm{Cu}$ & Vac. & $\mathrm{Ag}$ & $\mathrm{Vac}$. & $\mathrm{Au}$ & Vac. \\
\hline Benzene & 0.59 & 0.60 & 0.68 & 0.68 & 0.64 & 0.64 \\
Furan & 0.53 & 0.53 & 0.52 & 0.52 & 0.51 & 0.51 \\
Furan perp & 0.24 & 0.24 & 0.25 & 0.25 & 0.22 & 0.22 \\
Thiophene & 0.66 & 0.64 & 0.67 & 0.64 & 0.62 & 0.60 \\
Thiophene perp. & 0.40 & 0.38 & 0.40 & 0.37 & 0.25 & 0.23 \\
Thiophenol & 1.00 & 1.00 & 0.90 & 0.90 & 0.91 & 0.91 \\
Thiophenol perp. & 0.35 & 0.35 & 0.36 & 0.36 & 0.33 & 0.33 \\
Pyridine & 0.69 & 0.68 & 0.58 & 0.56 & 0.60 & 0.58 \\
Pyridine perp. & 0.86 & 0.86 & 0.66 & 0.66 & 0.64 & 0.64 \\
1,4-Benzenediamine (cis) & 1.17 & 1.14 & 1.04 & 1.03 & 1.10 & 1.09 \\
1,4-Benzenediamine (trans) & 1.24 & 1.24 & 1.09 & 1.09 & 1.12 & 1.12 \\
\hline Guanine & 1.07 & 1.17 & 1.03 & 1.09 & 0.94 & 0.99 \\
Cytosine & 0.96 & 1.03 & 0.90 & 0.94 & 0.77 & 0.81 \\
Adenine & 0.83 & 0.86 & 0.86 & 0.86 & 0.81 & 0.82 \\
Thymine & 0.67 & 0.72 & 0.69 & 0.72 & 0.67 & 0.70 \\
\hline
\end{tabular}




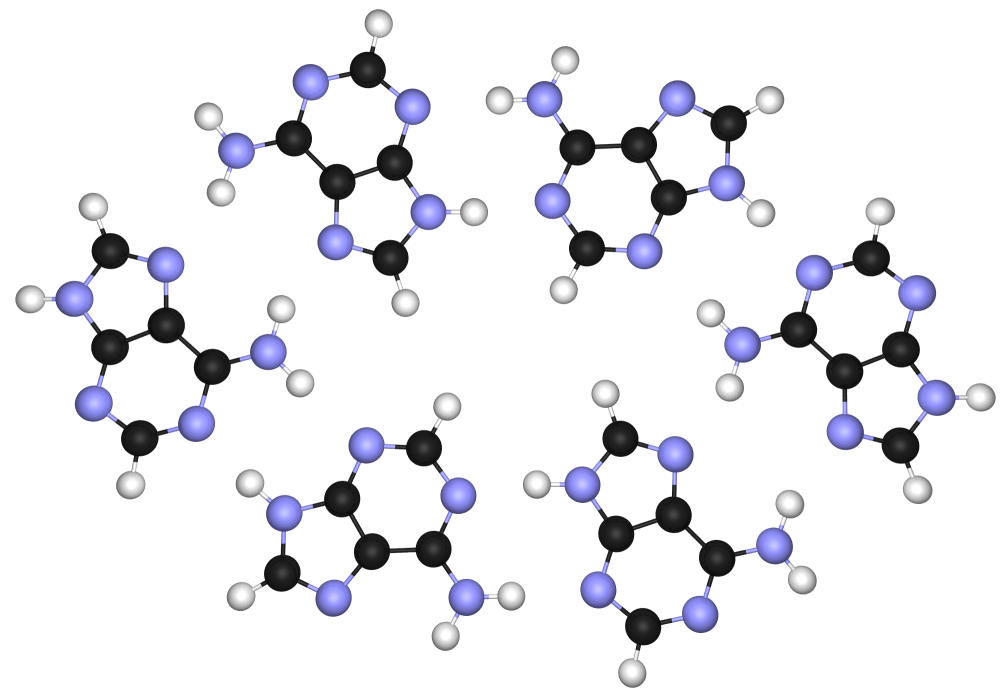

(a) adenine

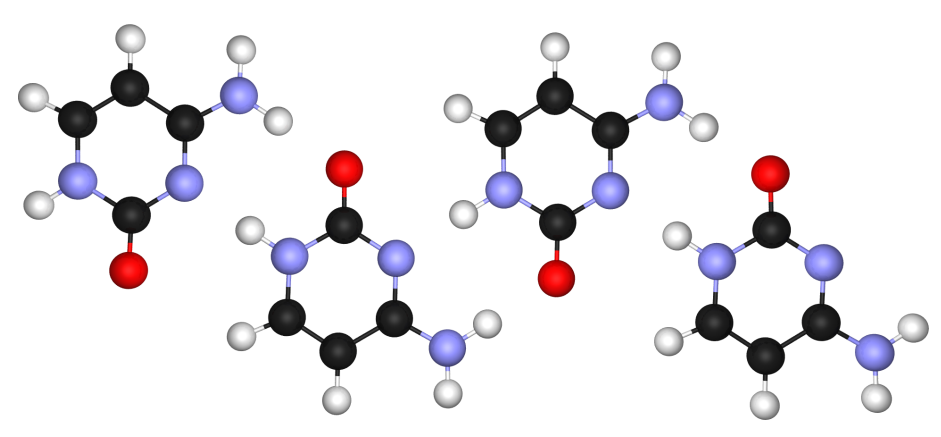

(c) cytosine

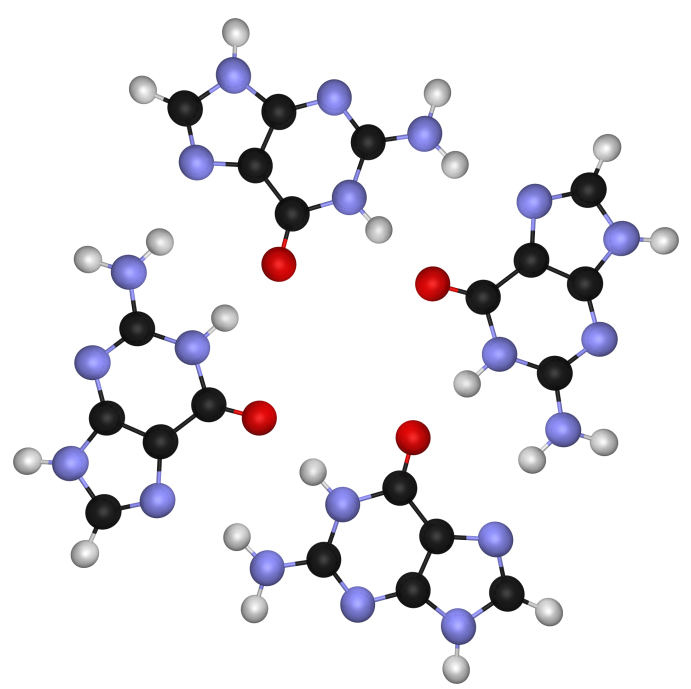

(b) guanine

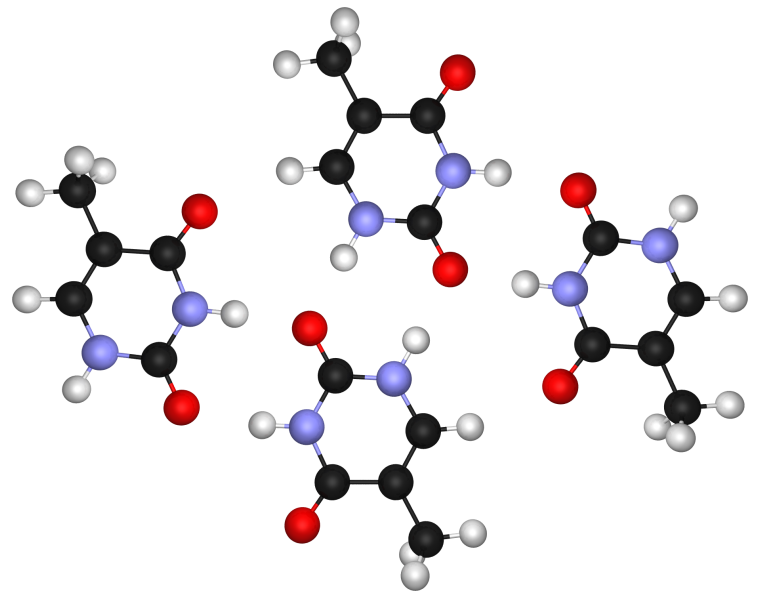

(d) thymine

Figure 1: Nucleobase clusters used to estimate the intermolecular hydrogen-bonding energies. 